\title{
Loss of prohormone convertase 2 promotes beta cell dysfunction in a rodent transplant model expressing human pro-islet amyloid polypeptide
}

\author{
Jaques A. Courtade ${ }^{1,2} \cdot$ Evan Y. Wang ${ }^{1,2} \cdot$ Paul Yen $^{1,2} \cdot$ Derek L. Dai ${ }^{1,2}$. \\ Galina Soukhatcheva $^{1,2} \cdot$ Paul C. Orban ${ }^{1,2}$ - C. Bruce Verchere ${ }^{1,2,3}$
}

Received: 4 May 2016 / Accepted: 3 October 2016 / Published online: 20 December 2016

(C) Springer-Verlag Berlin Heidelberg 2016

\begin{abstract}
Aims/hypothesis A contributor to beta cell failure in type 2 diabetes and islet transplants is amyloid formation by aggregation of the beta cell peptide, islet amyloid polypeptide (IAPP). Similar to the proinsulin processing pathway that generates insulin, IAPP is derived from a prohormone precursor, proIAPP, which requires cleavage by prohormone convertase (PC) $1 / 3$ and PC2 in rodent pancreatic beta cells. We hypothesised that loss of PC2 would promote beta cell death and dysfunction in a rodent model of human beta cell proIAPP overexpression.

Methods We generated an islet transplant model wherein immune-deficient mouse models of diabetes received islets expressing amyloidogenic human proIAPP and lacking PC2, leading to restoration of normoglycaemia accompanied by increased secretion of human proIAPP. Blood glucose levels were analysed for up to 16 weeks in transplant recipients and grafts were assessed for islet amyloid and beta cell number and death.

Results Hyperglycaemia (blood glucose $>16.9 \mathrm{mmol} / \mathrm{l}$ ) returned in $94 \%$ of recipients of islets expressing human
\end{abstract}

Electronic supplementary material The online version of this article (doi:10.1007/s00125-016-4174-2) contains peer-reviewed but unedited supplementary material, which is available to authorised users.

C. Bruce Verchere

bverchere@cfri.ca

1 Research Institute, BC Children's Hospital, 950 West 28th Avenue, Vancouver, BC V5Z 4H4, Canada

2 Department of Pathology and Laboratory Medicine, University of British Columbia, Vancouver, BC, Canada

3 Department of Surgery, University of British Columbia, Vancouver, BC, Canada
proIAPP and lacking PC2, whereas recipients of islets that express human proIAPP and normal PC2 levels remained normoglycaemic for at least 16 weeks. Islet graft failure was accompanied by a $\sim 20 \%$ reduction in insulin-positive cells, yet the degree of amyloid deposition and beta cell apoptosis was similar to those of controls expressing human proIAPP with functional PC2 levels.

Conclusions/interpretation PC2 deficiency in transplanted mouse islets expressing human proIAPP promotes beta cell loss and graft failure. Our data suggest that impaired $\mathrm{NH}_{2}-$ terminal processing and increased secretion of human proIAPP promote beta cell failure.

Keywords Beta cell · Islet amyloid · Islet transplant . Prohormone processing

\begin{tabular}{|c|c|}
\hline Abbreviations & \\
\hline $\mathrm{CHOP}$ & $\begin{array}{l}\text { CCAAT/enhancer-binding protein } \\
\text { homologous protein }\end{array}$ \\
\hline ER & Endoplasmic reticulum \\
\hline hproIAPP $P^{\mathrm{Tg} / 0}$ & $\begin{array}{l}\text { Hemizygous human pro-islet amyloid } \\
\text { polypeptide transgenic }\end{array}$ \\
\hline IAPP & Islet amyloid polypeptide \\
\hline $\mathrm{PC}$ & Prohormone convertase \\
\hline PDX1 & Pancreatic and duodenal homeobox 1 \\
\hline proIAPP & Pro-islet amyloid polypeptide \\
\hline proIAPP $_{1-48}$ & $\begin{array}{l}\mathrm{NH}_{2} \text {-terminally extended proIAPP } \\
\text { processing intermediate }\end{array}$ \\
\hline SCID & Severe combined immunodeficiency \\
\hline STZ & Streptozotocin \\
\hline TUNEL & $\begin{array}{l}\text { Terminal deoxynucleotidyl transferase } \\
\text { dUTP nick end labelling }\end{array}$ \\
\hline
\end{tabular}




\section{Introduction}

Islet amyloid creates pathological lesions in the pancreas of type 2 diabetic individuals [1]. The major component of amyloid is islet amyloid polypeptide (IAPP), a self-aggregating hormone, co-secreted with insulin from beta cells [2, 3]. Misfolded IAPP monomers assemble into oligomeric intermediates, which further generate amyloid fibres containing heparan sulphate proteoglycans, serum amyloid P and apolipoprotein E [4-6]. Prefibrillar IAPP species that arise during the transition from monomer to amyloid fibril elicit beta cell dysfunction and apoptosis [7-9], and provoke islet inflammation [9-11]. Cytotoxic IAPP aggregates mediate beta cell death and dysfunction in type 2 diabetes and in transplanted human islets $[10,12-14]$, although the mechanisms leading to cytotoxicity are poorly understood.

Adequate prohormone processing is vital to blood glucose homeostasis. Proinsulin processing is impaired in both type 2 diabetes [15-17] and islet transplants [18], resulting in elevated proinsulin:C-peptide ratios. Proinsulin processing involves cleavage by prohormone convertase (PC) $1 / 3$ and $\mathrm{PC} 2$ in order to generate insulin and C-peptide [17, 19]. In PC2-deficient mice, the absence of PC2 is partially compensated by the action of PC1/3 [20]; however, mice lacking PC2 still have elevated levels of proinsulin [21], a molecule with $10 \%$ bioactivity relative to insulin [22]. Similarly, in mice, pro-islet amyloid polypeptide (proIAPP) processing requires sequential action by $\mathrm{PC} 1 / 3$ and PC2 to generate IAPP [23, 24]. However, it is unclear whether impaired proIAPP processing plays a role in the pathogenesis of diabetes. Partially processed proIAPP species form aggregate structures in vitro $[25,26]$ and may be involved in amyloid formation and toxicity. Studying the effect of impaired prohormone processing has been limited by the lack of a suitable model, since global loss of PC2 results in developmental abnormalities [19, 27] and hypoglycaemia [20, 24].

Overexpression of human proIAPP in PC1/3- and PC2deficient cell lines promotes increased amyloid deposition and apoptosis [28]. We have previously demonstrated that cultured human proIAPP-overexpressing islets lacking PC2 develop increased amyloid formation and cell apoptosis compared with islets with normal PC2 levels [29]. We hypothesised that impaired beta cell proIAPP processing would further promote beta cell dysfunction in vivo. To test this hypothesis in a model of PC2 deficiency with adequate beta cell secretory drive, we transplanted mouse islets expressing human proIAPP but lacking PC2 into diabetic recipients and assessed graft function and amyloid formation.

\section{Methods}

Mice NOD.CB17-Prkdc $c^{\text {scid } / J ~(N O D / s e v e r e ~ c o m b i n e d ~ i m m u-~}$ nodeficiency [SCID]), C57BL/6J, FVB/N and FVB/N-
$\operatorname{Tg}($ Ins 2-IAPP)RHFSoel/J (human proIAPP transgenic) mice were purchased from the Jackson Laboratory (Bar Harbor, ME, USA). B6;129-Pcsk $2^{\mathrm{tm} 1 \mathrm{Dfs}} / \mathrm{J}$ ( $P c 2$ [also known as $P c s k 2]$-null) mice were generated as described previously [21]. All animals were housed in the animal facility of the Research Institute, BC Children's Hospital, in a $12 \mathrm{~h} / 12 \mathrm{~h}$ light/dark cycle, at $22 \pm 2{ }^{\circ} \mathrm{C}$, with free access to food and water. Male animals were used as islet donors and recipients.

$P c 2$-null mice were crossed with human proIAPP transgenic mice to generate hemizygous human proIAPP transgenic animals (hproIAPP ${ }^{\mathrm{Tg} / 0} \mathrm{Pc}^{+/-}$, herein referred to as proIAPP $\left.P^{+} P 2^{+}\right)$. These animals were crossed with $P c 2^{-/-}$mice to generate hybrid FVB; B6-hproIAPP ${ }^{\mathrm{Tg} / 0} P c 2^{-/-}$donors, herein referred to as proIAPP ${ }^{+} P c 2^{-}$mice, and additional control littermates (hproIAPP ${ }^{0 / 0} P c 2^{-/-}$and hproIAPP $P^{0 / 0} \mathrm{Pc}^{+/-}$mice, herein referred to as proIAPP $P^{-} P 2^{-}$and proIAPP $P c 2^{+}$, respectively).

For transplant experiments, islets were isolated from 16week-old mice fed breeder chow (9\% fat; Research Diets, New Brunswick, NJ, USA) from weaning. All animals were maintained according to the Canadian Council on Animal Care guidelines and experiments were approved by the University of British Columbia's Committee on Animal Care.

Islet isolation and transplantation Islets were isolated from 16-week-old donors as described in the electronic supplementary material (ESM) Methods. NOD/SCID recipients were administered a single dose $(180 \mathrm{mg} / \mathrm{kg})$ of streptozotocin (STZ; Sigma, St. Louis, MO, USA) and used for transplant if blood glucose was greater than $20 \mathrm{mmol} / \mathrm{l}$. Mice received 150 islets, which were transplanted into the left renal subcapsular space 4 days after STZ administration. Animals in which blood glucose levels did not normalise in the first week following transplant were removed from the study.

Western blot Islets were isolated from 16-week-old wild-type and $\mathrm{Pc}^{-/-}$animals for detection of glucagon and glucagon precursors, using a polyclonal guinea pig anti-mouse glucagon antibody (1:1000; ThermoFisher, Waltham, ME, USA), as described in ESM Methods.

Blood glucose monitoring Blood glucose was measured weekly using a OneTouch Ultra Glucometer (LifeScan, Burnaby, BC, Canada) via tail bleeds in islet donors (prior to islet isolation) and in islet transplant recipients. Graft failure was identified when fasting blood glucose levels were above $16.9 \mathrm{mmol} / \mathrm{l}$, at which point mice were euthanised.

Glucose and insulin tolerance tests To assess glucose tolerance, mice were fasted for $6 \mathrm{~h}$ and administered glucose i.p. ( $1.0 \mathrm{~g} / \mathrm{kg}$ body weight; Sigma). Glucose was measured by the OneTouch Ultra Glucometer (LifeScan) from tail bleeds 
at $0,15,30,60$ and 120 min following glucose administration. To assess insulin tolerance, mice were fasted for $6 \mathrm{~h}$ and administered $0.7 \mathrm{U} / \mathrm{kg}$ insulin i.p. (Novo Nordisk, Mississauga, ON, Canada), following which glucose measurements were obtained at $0,15,30,60$ and $120 \mathrm{~min}$. Mice were excluded from the experiment for humane reasons if blood glucose levels dropped below $1.8 \mathrm{mmol} / \mathrm{l}$.

\section{Plasma proinsulin, human proIAPP and IAPP measure-} ments At 6 weeks post transplantation, blood was collected via the saphenous vein in recipient mice. Plasma samples were isolated by centrifuging whole blood at $2000 \mathrm{~g} / 4^{\circ} \mathrm{C}$ and stored at $-80^{\circ} \mathrm{C}$ prior to analysis. Total proinsulin was measured using a rodent proinsulin ELISA (Mercodia, Uppsala, Sweden). Human IAPP and the $\mathrm{NH}_{2}$-terminally extended proIAPP processing intermediate (proIAPP ${ }_{1-48}$ ) were measured using an in-house ELISA which used antibody F002 (MedImmune, Gaithersburg, MD, USA) for capture, and either F064 (human proIAPP; MedImmune) or F025 (mature human IAPP; MedImmune) for detection on a Meso Sector S 600 plate reader (Meso Scale Discovery, Rockville, MD, USA).

Immunofluorescence staining Islet grafts were removed at 6 weeks post transplant, fixed overnight in paraformaldehyde (4\% wt/vol.) at $4{ }^{\circ} \mathrm{C}$ and stored in $70 \%$ (vol./vol.) ethanol prior to paraffin wax embedding and sectioning. Pancreatic sections $(5 \mu \mathrm{m})$ from mice age-matched to 16-week-old islet donors, and islet graft sections were dehydrated through a series of xylene/ethanol washes and blocked in $4 \%$ (vol./vol.) goat serum (Dako, Burlington, ON, Canada) for $1 \mathrm{~h}$. Sections were then stained with polyclonal guinea pig anti-insulin antibody (1:200; Dako) and rabbit anti-glucagon antibody (1:200; Abcam, Cambridge, UK) for $1 \mathrm{~h}$ at room temperature. Sections were incubated $(1 \mathrm{~h})$ with secondary antibodies, Alexa 594 goat anti-guinea pig (1:200; Invitrogen, Carlsbad, CA, USA) and Alexa 488 goat anti-rabbit (1:200; Invitrogen). Prior to CCAAT/enhancer-binding protein homologous protein $(\mathrm{CHOP})$ and pancreatic and duodenal homeobox 1 (PDX1) immunostaining, slides were incubated for $10 \mathrm{~min}$ at $95^{\circ} \mathrm{C}$ in $10 \mathrm{mmol} / 1$ citrate buffer for antigen retrieval. Immunostaining with CHOP (ThermoFisher, Burlington, ON, Canada) and PDX1 (Developmental Studies Hybridoma Bank, Iowa City, IA, USA) primary antibodies was performed overnight at $4^{\circ} \mathrm{C}$ using a 1:100 dilution of each antibody. Antibodies were validated by the provider, or where possible, on positive and negative control sections. For amyloid staining, sections were placed in $0.5 \%$ (wt/vol.) thioflavin $\mathrm{S}$ (Sigma) for $2 \mathrm{~min}$, following staining with secondary antibodies. Slides were washed twice with $70 \%$ (vol./vol.) ethanol and water prior to mounting. Images were obtained using an Olympus BX61 fluorescent microscope (Richmond Hill, ON, Canada) at $\times 20$ magnification. Cell death was measured via terminal deoxynucleotidyl transferase dUTP nick end labelling (TUNEL) staining, using the in situ cell death detection kit (Roche, Mississauga, ON, Canada).

Quantification of immunofluorescent staining Quantification of immunofluorescent staining was carried out on three representative sections from each group using an Image-Pro Plus Analyzer V6 (Olympus), as described in the ESM Methods. Where possible, experimenters were blind to outcome assessment. Graft beta cell area was determined as the insulin-positive area divided by the total insulin- and glucagon-positive (alpha cell) area and the proportion of TUNEL-positive beta cells was calculated as the number of TUNEL and insulin co-positive cells divided by total insulinpositive cells. Amyloid area was calculated as the thioflavin S positive area divided by total graft area and amyloid severity (\%) was calculated as total amyloid area divided by total islet area $(\times 100)$, while per cent amyloid prevalence was calculated as number of islets containing amyloid divided by total number of islets $(\times 100)$.

Statistical analyses Data are expressed as mean \pm SEM. Differences between two groups were determined using Student's $t$ test, while differences among several groups were analysed by one-way ANOVA followed by Tukey's post hoc test for multiple comparisons. Statistical analyses were performed using GraphPad Prism 5.0 software (GraphPad, La Jolla, CA, USA). Values were considered significant if $p<0.05$.

\section{Results}

Lack of PC2 promotes mild hypoglycaemia and improved glucose tolerance

To test whether elevated human proIAPP levels promote beta cell failure, we generated mice with beta cell expression of human proIAPP and lacking PC2 (proIAPP $P^{+} \mathrm{C}^{-}$), as well as control mice with human proIAPP and one $P c 2$ allele $\left(\right.$ proIAPP $P^{+} \mathrm{PC}^{+}$) (Fig. 1a). We generated additional control groups of mice that did not express human proIAPP but lacked PC2 (proIAPP $P^{-} 2^{-}$), as well as mice that did not express human proIAPP and had normal PC2 function (proIAPP $P c 2^{+}$) (Fig. 1a). $P c 2^{+/-}$mice were used instead of $P c 2^{+/+}$mice for ease of breeding.

We first determined whether global PC2 deficiency had an impact on glucose homeostasis, with and without expression of the human proIAPP transgene. $\mathrm{FVB} ; \mathrm{B} 6$ hybrid proIAPP $^{-} P \mathrm{C}^{+}$ mice tended to have higher body weight on breeder chow, relative to other genotypes, although this was not statistically significant (Fig. 1b). None of the four genotypes were hyperglycaemic, although mice lacking PC2 demonstrated mild 
a

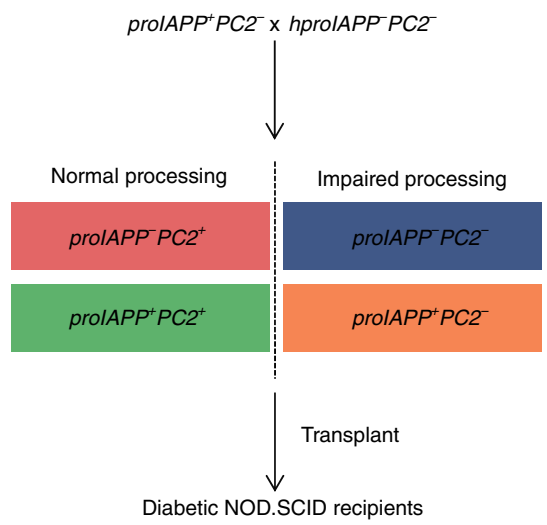

Fig. 1 Hypoglycaemia and improved glucose tolerance in PC2-deficient mice. (a) Diagram of breeding scheme used to generate study animals. (b) Body weight of islet donors at 16 weeks of age. (c) i.p. glucose tolerance test in 16-week-old donor animals. Data are expressed as mean \pm SEM. Red bar, and red circles and solid red line, $\operatorname{proIAPP}^{-} P c 2^{+}(n=4$ mice); blue bar, and blue circles and solid blue line, $\operatorname{proIAPP}^{-} P c 2^{-}(n=6$ mice);

hypoglycaemia (Fig. 1c), likely related to their inability to produce mature glucagon [20]. Lack of mature glucagon production in PC2-deficient animals was demonstrated by western blot (ESM Fig. 1). Glucose tolerance was improved in PC2deficient compared with mice with PC2 expression (Fig. 1c), as previously reported in PC2-deficient mice fed normal chow [20]. We observed a mild difference in glucose tolerance between proIAPP $^{+} P c 2^{+}$and proIAPP $P c 2^{+}$mice at 16 weeks of age, suggesting that expression of the human proIAPP transgene promotes early beta cell dysfunction in our model.

\section{ProIAPP $^{+} \mathrm{Pc}^{-}$mice have abnormal islet morphology and reduced amyloid formation}

To determine whether proIAPP ${ }^{+} P C 2^{-}$mice have changes in islet morphology, we determined the proportion of beta to alpha cells in these animals. Mice lacking PC2 had an increased proportion of alpha cells, such that the ratio of beta:alpha cells was $\sim 1: 1$ (Fig. 2a-c), as reported previously [20]. Interestingly, despite this abnormal islet architecture, proIAPP $P c 2^{-}$and proIAPP $P^{+} P c 2^{-}$ donors had similar numbers of beta cells per islet compared with controls expressing Pc2 (ESM Fig. 2). Staining of pancreatic sections with thioflavin $\mathrm{S}$ revealed small, punctate amyloid deposits in islets of proIAPP $P^{+} \mathrm{Pc}^{+}$mice but not in those of proIAPP $P^{+} P c 2^{-}$mice (Fig. 2c-e). The lack of detectable amyloid in mice expressing human proIAPP and lacking PC2 is likely related to these animals being mildly hypoglycaemic, resulting in a low drive for proIAPP synthesis [30].

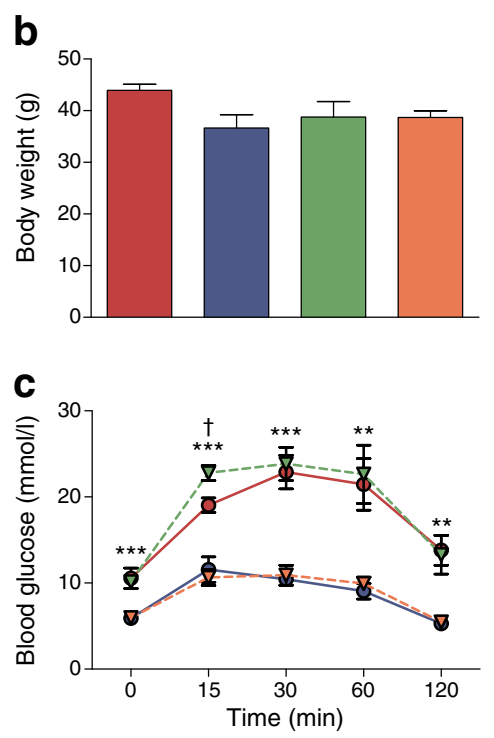

green bar, and green triangles and dashed green line, proIAPP $P^{+} 2^{+}$ ( $n=6$ mice); orange bar, and orange triangles and dashed orange line, proIAPP $P^{+} P c 2^{-}$( $n=6$ mice). $* * p<0.01$, *** $p<0.001$, proIAPP $P c 2^{+}$ vs proIAPP $P c 2^{-}$donors; $\uparrow p<0.05$, proIAPP $P^{+} P c 2^{+}$vs proIAPP $P c 2^{+}$ donors

\section{Loss of PC2 and overexpression of human proIAPP lead to rapid failure of islet transplants}

Since PC2-deficient mice tend to be hypoglycaemic because of their inability to make mature glucagon, to test whether loss of PC2 promotes amyloid formation and beta cell dysfunction in vivo we transplanted islets from these mice into STZ-induced diabetic, immune-deficient (NOD.SCID) recipients. Recipient mice were predicted to have normal glucagon levels but severely reduced beta cell numbers following STZ administration. We chose islet donors with minimal amyloid deposition and normal glucose tolerance to enable us to investigate differences in phenotype that arise following transplantation. Following islet transplantation and initial normalisation of blood glucose, non-fasting blood glucose levels steadily increased in mice transplanted with islets from proIAPP ${ }^{+} P c 2^{-}$mice (Fig. 3a). By contrast, we observed no changes in blood glucose levels in recipients of proIAPP $P^{+} P 2^{+}$, proIAPP $^{-} P c 2^{-}$or proIAPP $P^{-} \mathrm{C}^{+}$islet grafts. Strikingly, in a cohort of islet graft recipients followed for 16 weeks, 15 of 16 recipients of proIAPP $P^{-} 2^{-}$islet transplants became hyperglycaemic (blood glucose $>16.9 \mathrm{mmol} / \mathrm{l}$ ), whereas all recipients of control islet grafts remained normoglycaemic (Fig. 3b). These results suggest that loss of PC2 promotes beta cell failure in a model of human proIAPP overexpression.

We next examined whether islet graft failure was accompanied by amyloid formation and beta cell loss. To determine whether islet graft failure was due to loss of beta cells shortly 
Fig. 2 Islet histology in mice expressing human proIAPP and lacking PC2. (a) Insulin and glucagon immunostaining of pancreatic sections from donor animals. (b) Ratio of beta cell:islet area and (c) ratio of alpha cell:islet area (islet area $=$ total alpha cell plus beta cell area). (d) Insulin and amyloid staining in donor islets. White arrows indicate the location of amyloid deposits. (e) Quantification of amyloid severity as determined by amyloid area relative to total islet graft area. (f) Quantification of amyloid prevalence as determined by the number of islets containing amyloid relative to the total number of islets. Scale bars, $100 \mu \mathrm{m}$. Data are expressed as mean \pm SEM. Red bars, proIAPP $P c 2^{+}(n=4$ mice); blue bars, proIAPP $P c 2$ ( $n=6$ mice); green bars, proIAPP $P^{+} \mathrm{C} 2^{+}$( $n=6$ mice); orange bars, proIAPP $P^{+} P 2^{-}$ ( $n=6$ mice). ${ }^{* *} p<0.01$, $* * * p<0.001$

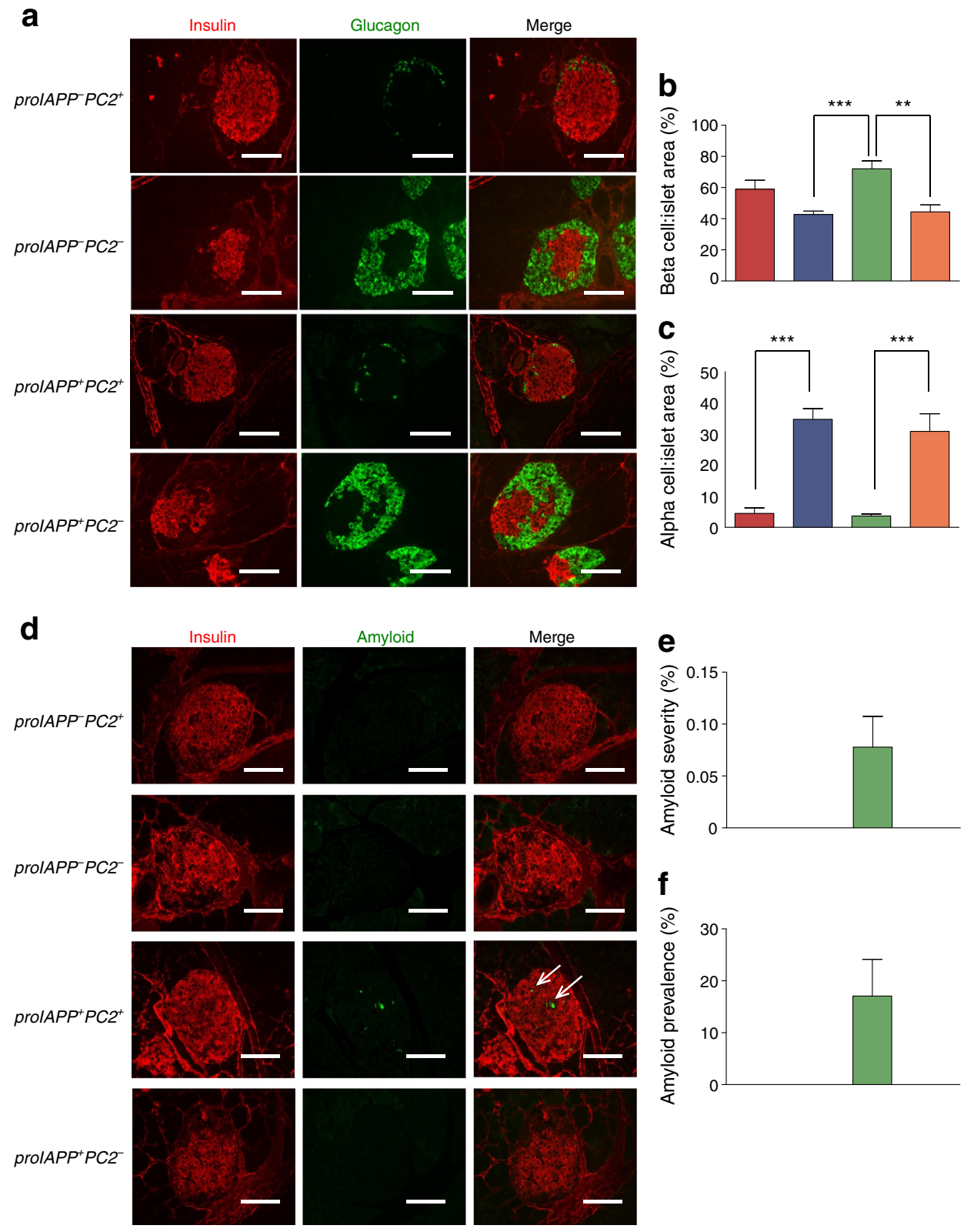

following transplantation, we quantified the number and proportion of insulin-positive cells in islet grafts at 1 and 6 weeks post transplant. At 1 week post transplantation, the proportion of insulin-positive cells in proIAPP $P^{+} P 2^{-}$transplants tended to be reduced (although non-significantly) compared with proIAPP ${ }^{+} \mathrm{Pc}_{\mathrm{C}}{ }^{+}$grafts, whereas the proportion of glucagon-positive cells was significantly elevated in proIAPP $P^{-} P 2^{-}$(compared with proIAPP $P c 2^{+}$) and proIAPP ${ }^{+} P c 2^{-}$(compared with proIAPP ${ }^{+} P c 2^{+}$) islet grafts (Fig. $4 \mathrm{a}-\mathrm{c}$ ). The proportion of insulin-positive cells in proIAPP $P^{+} P 2^{-}$was further reduced compared with proIAPP $P^{+} P 2^{+}$islet transplants compared at 6 weeks post transplant, making this finding significant (Fig. 4d, e). The number of insulin-positive cells per islet 1 week post transplant was similar in proIAPP $P^{+} P 2^{-}$and proIAPP $P^{+} P c 2^{+}$islet grafts (ESM Fig. 3a) but significantly lower in proIAPP ${ }^{+} P C 2^{-}$at 6 weeks post transplant (ESM Fig. 3b). These data suggest that most beta cell loss in this model does not occur immediately following transplantation, but rather over several weeks post transplant, possibly as IAPP toxicity accumulates. The proportion of graft area comprising alpha cells was non-significantly higher in proIAPP ${ }^{-} P C 2^{-}$compared with proIAPP $P c 2^{+}$islets at 1 week post transplantation (Fig. 4d, e), but by 6 weeks post transplant, the alpha cell proportion had returned to similar levels as those of proIAPP ${ }^{-} P 2^{+}$islet grafts. Thus, the alpha cell hyperplasia associated with $\mathrm{PC} 2$ deficiency was normalised following transplantation of islets into recipients with normal glucagon production. Somewhat surprisingly, thioflavin S 


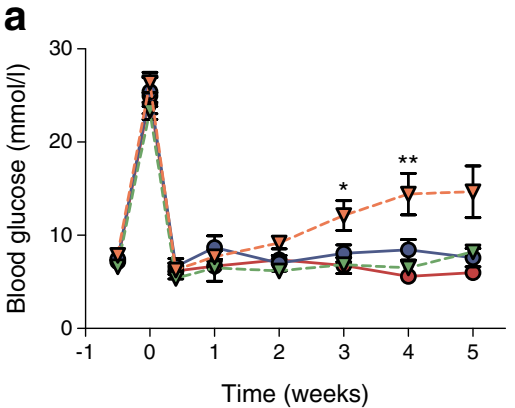

Fig. 3 Islet graft failure in recipients of transplanted islets expressing human proIAPP and lacking PC2. (a) Weekly non-fasting blood glucose measurements in transplant recipients. Red circles and solid red line, proIAPP $P^{-} \mathrm{Pc}^{+}(n=6$ mice $)$; blue circles and solid blue line, proIAPP $P c 2^{-}(n=5$ mice $)$; green triangles and dashed green line, proIAPP $P^{+} P c 2^{+}(n=7$ mice); orange triangles and dashed orange line, proIAPP $P^{+} P 2^{-}$( $n=8$ mice). (b) Survival graph displaying per cent of

staining revealed a similar degree of amyloid deposition in proIAPP ${ }^{+} P c 2^{-}$compared with proIAPP ${ }^{+} P c 2^{+}$islet grafts (Fig. 4f, g). While these data do not support amyloid as the major cause of failure of proIAPP $P c 2^{-}$islet transplants, it remains possible that non-detectable, prefibrillar amyloid aggregates contributed to graft failure in this model. TUNEL staining revealed no significant differences in islet cell death at 6 weeks post transplant among any of the groups (Fig. 4h, i).

\section{Elevated proinsulin and human proIAPP levels are associated with early islet graft failure}

To better characterise the effect of PC2 loss on prohormone processing in this model, we measured plasma concentrations of mouse proinsulin, human proIAPP ${ }_{1-48}$ and mature human IAPP. As expected, at 6 weeks post transplantation, recipients of grafts lacking PC2 had markedly elevated circulating proinsulin levels (Fig. 5a). By contrast, mouse C-peptide concentrations were similar among all groups (Fig. 5b). Our finding that C-peptide levels are similar in recipients of $\mathrm{Pc}^{-/}$and $\mathrm{Pc}^{+/-}$islet grafts is somewhat surprising, and may arise from compensatory increases in proinsulin production and/or PC1/ 3 synthesis in PC2-deficient beta cells. Circulating levels of the human proIAPP processing intermediate, proIAPP $_{1-48}$, were elevated in mice that received proIAPP $P^{+} P c 2^{-}$islets compared with recipients of proIAPP $P^{+} P 2^{+}$islets (Fig. 5 c), while mature human IAPP levels also tended to be increased in recipients of proIAPP $P^{+} P c 2^{-}$islets $(p=0.054$; Fig. $5 \mathrm{~d})$. Since proIAPP $P c 2^{-}$islet transplants did not fail during the 16 week study period, our data suggest that elevated proinsulin levels alone are insufficient to promote graft failure; however, the combination of increased proinsulin and proIAPP ${ }_{1-48}$ production by proIAPP ${ }^{+} P 2^{-}$islet grafts appears sufficient for rapid transplant failure.

We performed insulin tolerance tests in recipients 6 weeks post transplant to ascertain whether transplant failure might be b

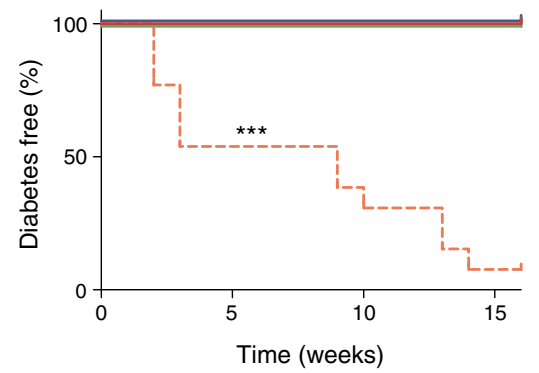

recipients with blood glucose levels below $16.9 \mathrm{mmol} / \mathrm{l}$. Red solid line, proIAPP $P c 2^{+}$( $n=6$ mice); solid blue line, $\operatorname{proIAPP}^{-} P c 2^{-} \quad(n=5$ mice); solid green line, proIAPP $P^{+} P 2^{+}(n=8$ mice $)$; dashed orange line, proIAPP $P^{+} P 2^{-}(n=13$ mice $)$. Data are expressed as mean $\pm \mathrm{SEM}$. ${ }^{*} p<0.05, * * p<0.01, * * * p<0.001$, proIAPP $P^{+} P c 2^{-}$vs all other islet recipients

attributed to changes in insulin sensitivity. Compared with mice with proIAPP ${ }^{+} P c 2^{+}$grafts, recipients of proIAPPP $P c 2^{-}$ islets had significantly higher blood glucose levels during the first $30 \mathrm{~min}$ of the test (Fig. 5e) and a greater change in blood glucose levels at 60 and $120 \mathrm{~min}$ (Fig. 5f). These data imply that animals with failing proIAPP $P^{+} P 2^{-}$grafts were, if anything, more insulin sensitive, and suggest that hyperglycaemia was a result of impaired graft function and not peripheral insulin resistance. One explanation for the improved insulin sensitivity in recipients of proIAPP $P^{+} P 2^{-}$grafts, despite the presence of hyperglycaemia, could be related to bioactivity of the very high circulating proinsulin levels in these mice (Fig. 5a).

\section{Gene and protein expression and ER stress analysis in proIAPP $P^{+} 2^{-}$islet grafts}

To gain insight into the mechanism underlying failure of proIAPP ${ }^{+} P c 2^{-}$islet transplants, we assessed gene expression in grafts 6 weeks post transplantation. $P d x 1$ and insulin (Ins2) mRNA tended to be lower in proIAPP ${ }^{+} P c 2^{-}$grafts compared with proIAPP ${ }^{+} P c 2^{+}$grafts, although, due to high variance and small sample size, these changes were not statistically significant (Fig. 6a). Immunostaining data supported our gene expression findings: the intensity of PDX1 immunostaining appeared reduced in proIAPP $P^{+} \mathrm{P}^{-}$grafts compared with controls (Fig. 6b). Coinciding with this effect, there was a reduction in PDX1-positive beta cell per cent in proIAPP $P^{+} \mathrm{C}^{-}$ grafts (Fig. 6c), suggesting that graft failure in this model is accompanied by reduced PDX1 production in beta cells. The absence of PDX1 expression in non-insulin-producing cells (Fig. 6b), as well as the lack of any observable insulin and glucagon co-staining (Fig. 4d), suggested that there were no changes in pancreatic progenitor cells or occurrence of trans-differentiation at 6 weeks post transplant. We also examined CHOP levels as a late-stage marker of endoplasmic 

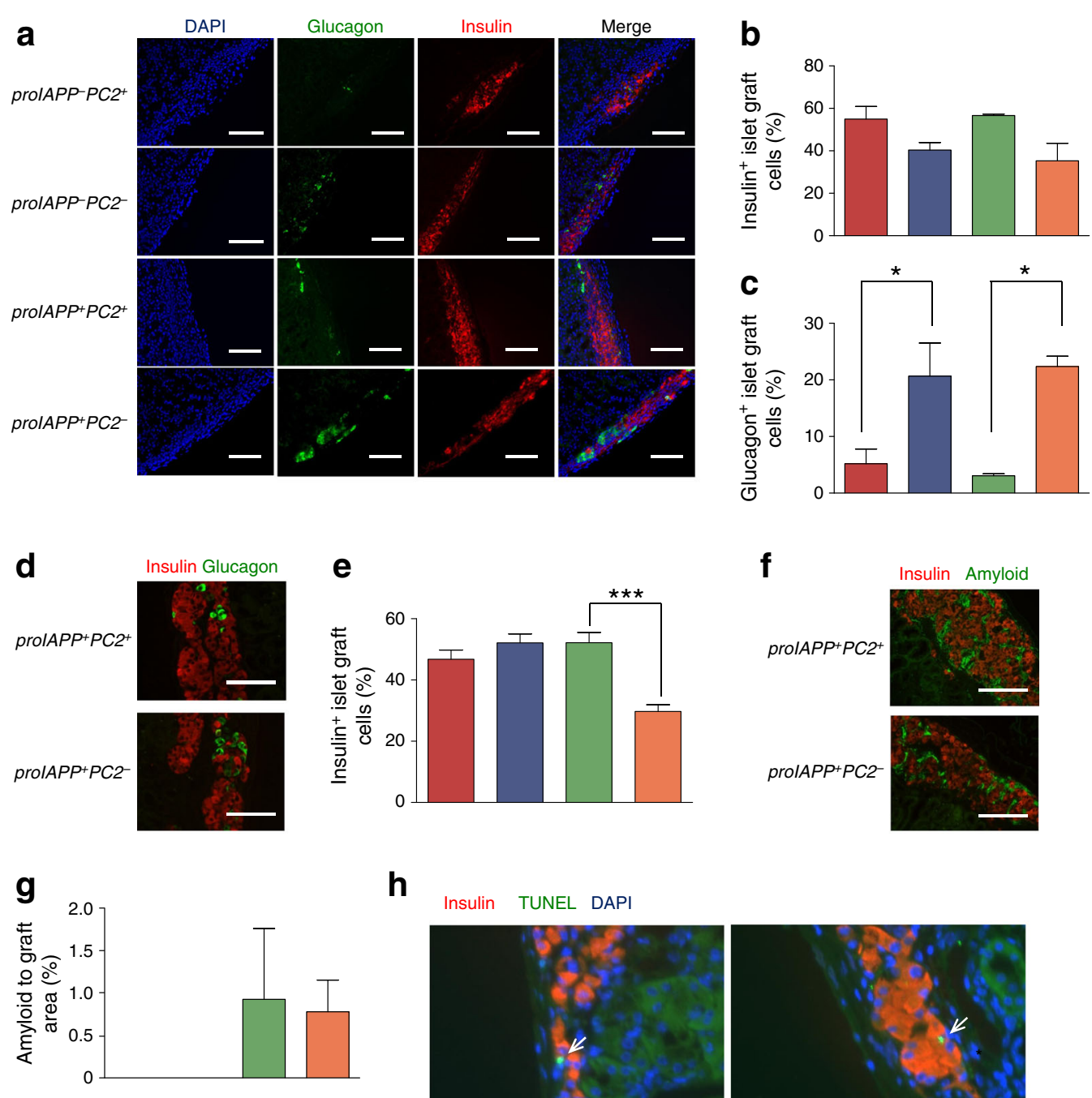

h

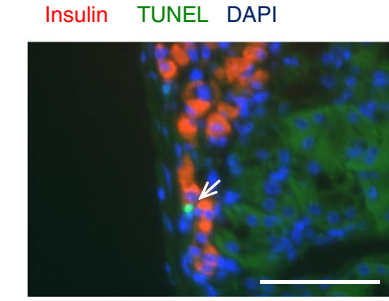

prolAPP $+P C 2^{+}$

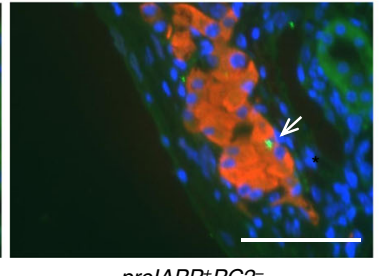

prolAPP ${ }^{+} P C 2^{-}$

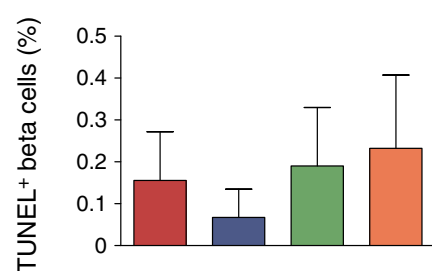

Fig. 4 Islet histology in recipients of islets expressing human proIAPP and lacking PC2. (a) DAPI, glucagon and insulin immunostaining, (b) quantification of insulin-positive graft cells and (c) glucagon-positive graft cells 1 week post transplant. (d) Insulin and glucagon staining and (e) quantification of insulin-positive islet graft cells 6 weeks post transplant. (f) Histological staining of amyloid deposits and (g) quantification of amyloid severity calculated as amyloid area relative to total graft area. (h) Insulin, TUNEL and DAPI staining (white arrows denote TUNELpositive beta cells) and (i) quantification showing the extent of TUNEL positivity in 6-week-old recipients. Red bars, proIAPP $P^{-} 2^{+}$; blue bars,

reticulum (ER) stress-induced apoptosis in failing islet grafts. None of the recipient groups' beta cells stained positive for CHOP (Fig. 6d, ESM Fig. 4), suggesting that ER stress-induced apoptosis was not a characteristic of islet graft failure in this model, at least at 6 weeks post transplant.
proIAPP $P_{C 2}^{-}$; green bars, proIAPP $P^{+} P c 2^{+}$; orange bars, proIAPP $P^{+} P 2^{-}$. For $(\mathbf{a}-\mathbf{c})$ : proIAPP $P^{-} \mathrm{P}^{+}, n=5$ mice; proIAPP $P^{-} P c 2^{-}, n=5$ mice; proIAP $P^{+} P 2^{+}, n=4$ mice; proIAPP $P^{+} P 2^{-}, n=3$ mice. For (d) and (e): proIAPP $P c 2^{+}, n=7$ mice; proIAPP $P c 2^{-}, n=6$ mice; proIAPP $P^{+} P c 2^{+}$, $n=7$ mice; proIAPP $P^{+} P c 2^{-}, n=8$ mice. For (f) and (g): proIAPP $P c 2^{+}$, $n=4$ mice; proIAPP $P^{-} P c 2^{-}, n=4$ mice; $\operatorname{proIAPP} P^{+} P c 2^{+}, n=5$ mice; proIAPP $P^{+} P c 2^{-}, n=6$ mice. For (h) and (i): proIAPP $P c 2^{+}, n=7$ mice; proIAPP $P^{-} 2^{-}, n=6$ mice; proIAPP $P^{+} P c 2^{+}, n=7$ mice; proIAPP ${ }^{+} P c 2^{-}$, $n=8$ mice. Data are expressed as mean \pm SEM. Scale bars, $100 \mu \mathrm{m}$. $* p<0.05, * * * p<0.001$

Our own previous data demonstrated that human IAPP aggregates induce inflammation in islet transplants [9]. Islets from proIAPP $P^{+} \mathrm{C}^{-}$donors displayed a trend towards increased $C d 11 b$ (also known as Itgam) expression compared with the other groups (Fig. 6e); however, no difference was 

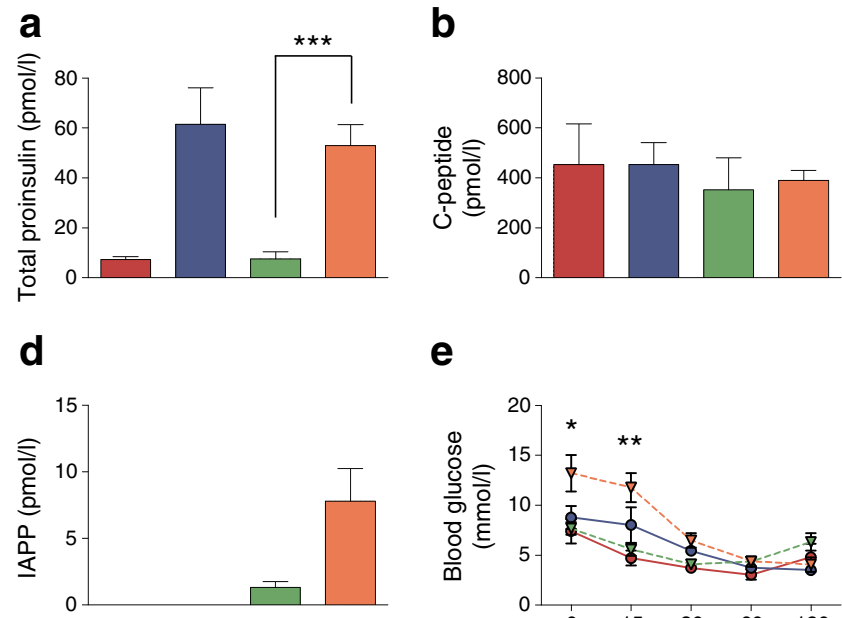

e
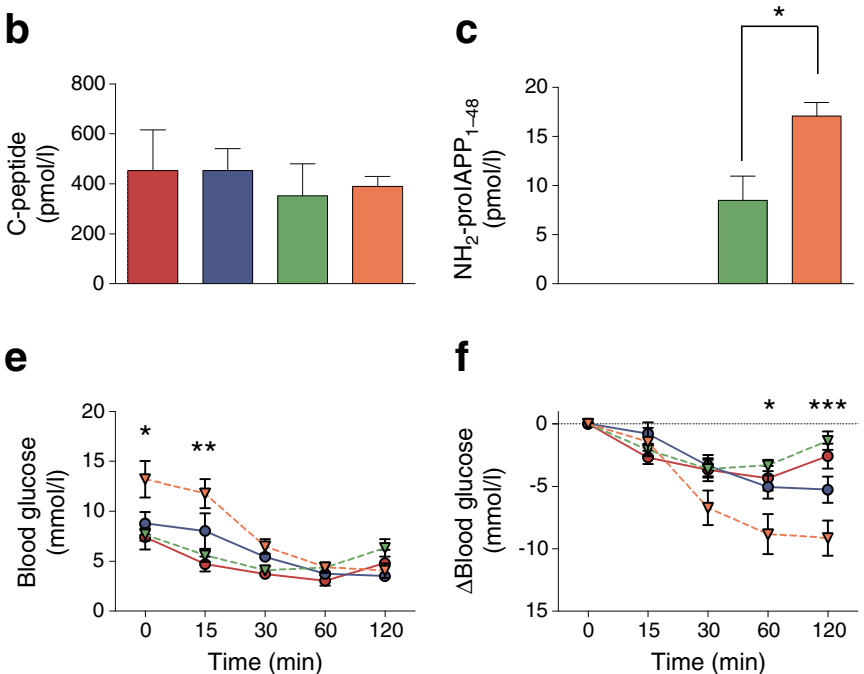

$f$

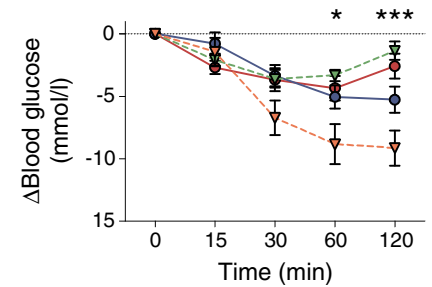

Fig. 5 Elevated proinsulin and human proIAPP ${ }_{1-48}$ levels in recipients of transplanted islets expressing human proIAPP and lacking PC2. (a) Total plasma proinsulin, (b) C-peptide, (c) $\mathrm{NH}_{2}$ - rroIAPP $_{1-48}$ and (d) mature IAPP concentrations in recipients 6 weeks post transplant. For (a-d): red bars, proIAPP $P c 2^{+}$( $n=4$ mice); blue bars, proIAPP $P c 2^{-}$( $n=4$ mice); green bars, proIAPP $P^{+} P 2^{+}\left(n=5\right.$ mice); orange bars, proIAPP $P^{+} P c 2^{-}(n=6$ mice). $(\mathbf{e}, \mathbf{f})$ Insulin tolerance tests at 6 weeks post transplant presented as (e) blood

apparent in the expression of $I l 1 b, N l r p 3$ or $T l r 2$ in this model. There was also a trend towards decreased expression of both Pcsk1, necessary for cleavage of the C-terminal end of proIAPP, as well as its endogenous inhibitor Pcsk1n, in recipients of proIAPP $P^{+} P 2^{-}$islet grafts (Fig. 6f). We also observed a trend towards downregulation of Sgnel (also known as Scg5), which codes for the PC2 chaperone, 7B2, and Cpe, responsible for removal of paired basic residues following PC-mediated cleavage (Fig. 6f). These data demonstrate the possibility that further impairments in prohormone processing may accompany declining islet graft function in this model.

\section{Discussion}

Identification of factors that limit beta cell viability is vital to the understanding of diabetes development. Here, we demonstrate that dysregulation of human proIAPP processing leads to premature beta cell failure in an islet transplant model. Although the degree of amyloid deposition was similar between mice with and without expression of PC2, deficiency of this enzyme in the presence of human proIAPP expression led to significant loss of beta cells. The data suggest a model in which impaired prohormone processing promotes beta cell failure.

The toxic species associated with IAPP aggregation remains elusive, although prefibrillar, oligomeric species appear to be most deleterious for beta cell survival [7, 9]. Here, we examined whether overexpression of human proIAPP in a model lacking PC2 production exacerbated beta cell failure. glucose concentration and (f) change in blood glucose concentration $(\mathrm{mmol} / \mathrm{l})$. For $(\mathbf{e})$ and $(\mathbf{f})$ : red circles and solid red line, proIAPP $P c 2^{+}$ ( $n=7$ mice); blue circles and solid blue line, proIAPP $P c 2^{-}(n=8$ mice); green triangles and dashed green line, $\operatorname{proIAPP}^{+} P c 2^{+}(n=6$ mice); orange triangles and dashed orange line, proIAPP $P^{+} P 2^{-}(n=9$ mice $)$. Data are expressed as mean \pm SEM. $* p<0.05, * * p<0.01, * * * p<0.001$, proIAPP $P^{+} 2^{-}$vs proIAPP $P c 2^{+}$islet recipients

Surprisingly, even with rapid islet graft failure and a $20 \%$ reduction in insulin-positive cells in islet transplants expressing human proIAPP and lacking PC2, we were unable to detect increases in amyloid deposition or beta cell apoptosis. These observations are in contrast with our previous in vitro findings [29]. Mature amyloid fibrils appear to be less deleterious to beta cells than prefibrillar species and improvements in glucose tolerance do not always correlate with reduced amyloid formation [10]. Thus, while our data do not support the hypothesis that impaired $\mathrm{NH}_{2}$-terminal processing of proIAPP leads to amyloid formation in vivo, it remains plausible that increased production of prefibrillar proIAPP aggregates are present in PC2-deficient, human proIAPP-expressing islet grafts and are inducing beta cell dysfunction and death, but that these are not detectable by thioflavin S.

Although aggregation of proIAPP forms has not been studied as extensively as in mature IAPP, it has been reported that proIAPP species aggregate in the presence of negatively-charged artificial membranes [31]. The positively-charged $\mathrm{NH}_{2}$-terminus of unprocessed proIAPP interacts with heparin, possibly enabling attachment to heparan sulphate proteoglycans on islet basement membranes and forming a nidus for amyloid development [6, 32, 33]. Whether this process impacts other signalling pathways or is a contributor to beta cell apoptosis has not been examined. Although preliminary, our gene expression analysis suggests that accumulation of human proIAPP species may compromise beta cell differentiation and function, and prohormone processing, and may induce pro-inflammatory pathways. However, a more thorough analysis at the protein level will 
a

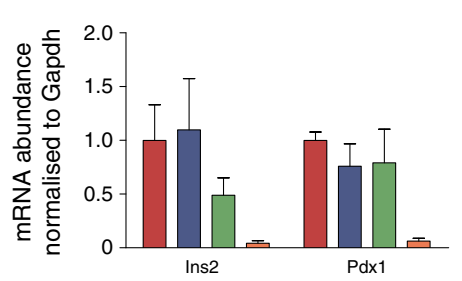

C

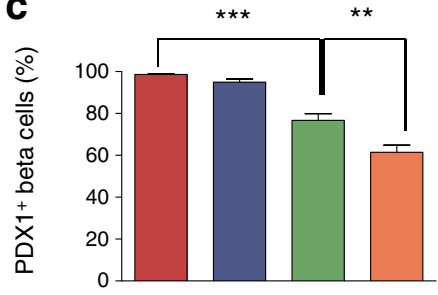

\section{b}

prolAPP-PC2

prolAPP+PC2+

prolAPP $+P C 2$
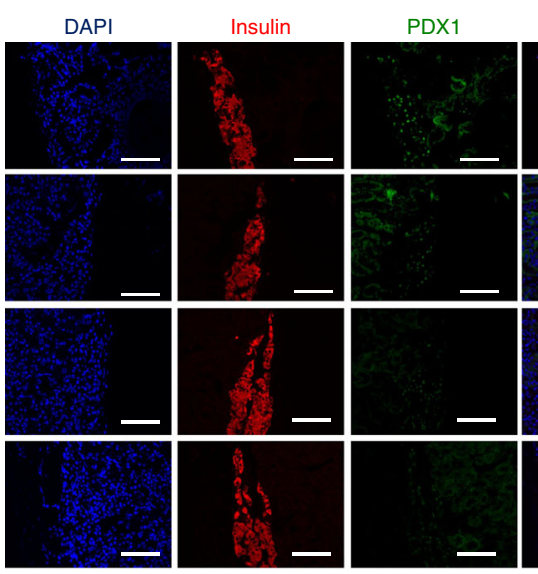

Merge

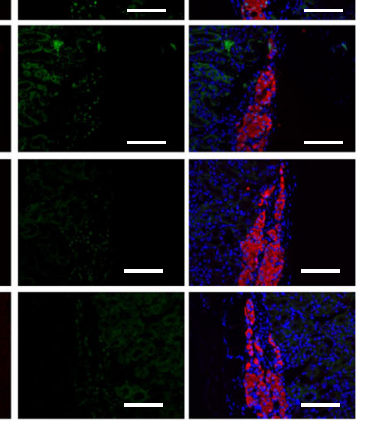

d

proIAPP-PC2

DAPI

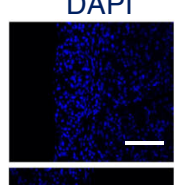

Insulin

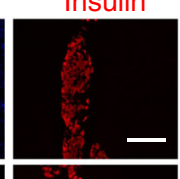

CHOP

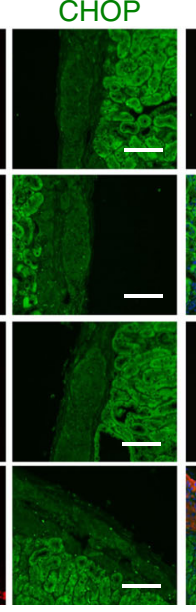

Merge

prolAPP-PC2-

prolAPP $+P C 2^{+}$

prolAPP $+P C 2$

\section{e}

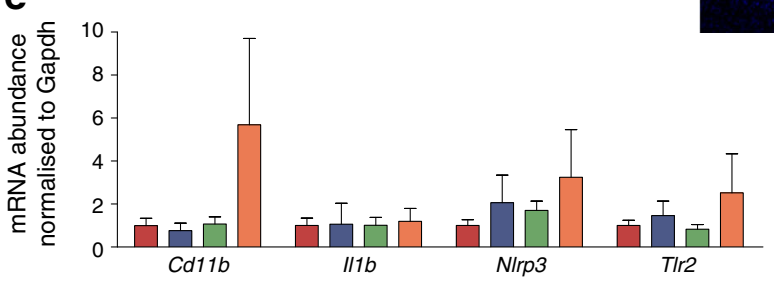

$\mathbf{f}$

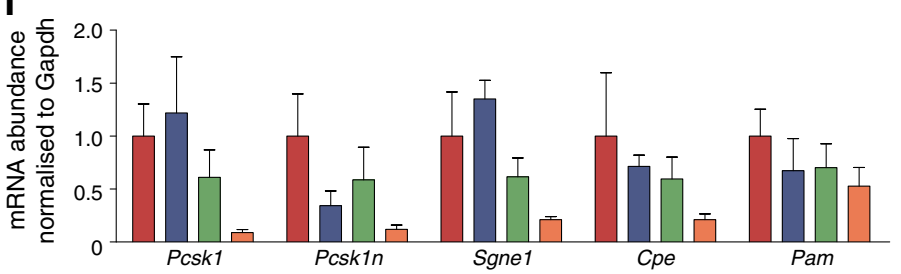

Fig. 6 Gene expression and immunostaining for PDX1 and CHOP in transplanted islets expressing human proIAPP and lacking PC2. (a) Quantitative real-time PCR (qPCR) analysis of beta cell markers, Ins2 and $P d x 1$. (b) DAPI, insulin and PDX1 staining in islet graft recipients. (c) Quantification of PDX1-positive beta cells in islet grafts. (d) DAPI, insulin and CHOP staining in islet graft recipients. (e) qPCR analysis of pro-inflammatory genes and (f) qPCR analysis of prohormone processing genes. Relative mRNA levels for each transcript were determined as the fold increase over Gapdh. Red bars, proIAPP $P_{c}^{-} 2^{+}$; blue bars,
proIAPP $P_{c}^{-} 2^{-}$; green bars, proIA $P^{+} P c 2^{+}$; orange bars, proIAPP $P^{+} P 2^{-}$. For (a), proIAPP $P^{-} P c 2^{+}, n=6$ mice; proIAPP $P^{-} P c 2^{-}, n=5$ mice; proIAPP $P^{+} P 2^{+}, n=6$ mice; proIAPP $P^{+} P c 2^{-}, n=5$ mice. For $(\mathbf{b}-\mathbf{d})$ : proIAPP $P c 2^{+}, n=7$ mice; proIAPP $P c 2^{-}, n=6$ mice; proIAPP $P^{+} P c 2^{+}$, $n=7$ mice; proIAPP $P^{+} P 2^{-}, n=8$ mice. For (e) and (f): proIAPP $P c 2^{+}$, $n=6$ mice; proIAPP $P_{c}^{-} 2^{-}, n=5$ mice; proIAPP $P^{+} P c 2^{+}, n=6$ mice; proIAPP $P c 2^{-}, n=5$ mice. Data are expressed as mean \pm SEM. Scale bars, $100 \mu \mathrm{m} . * * p<0.01, * * * p<0.001$

Pc2-null grafts expressing human proIAPP suggests ER stress was not an important contributor to graft failure in this model. 
A previous study revealed that human IAPP expression led to islet transplant failure 2 weeks following transplantation of a suboptimal mass of 100 islets [13]. Because our transplant studies had the additional stress of impaired human proIAPP and proinsulin processing, we chose to transplant a more optimal mass of 150 islets. Islets from PC2-deficient mice showed alpha cell hyperplasia ( 1:1 ratio of beta cells:alpha cells) compared with islets from mice with normal PC2 expression. Despite this abnormal islet morphology, transplanted islets lacking PC2 and without human proIAPP expression remained functional. Immunostaining revealed restoration of normal beta and alpha cell histology in PC2-deficient grafts at 6 weeks post transplant, in keeping with other findings that alpha cell hyperplasia is driven by lack of glucagon production [34]. To avoid potentially confounding effects of the abnormal islet morphology in Pc2-null mice, as well as the limitations of islet transplant models, future studies should examine amyloid formation and beta cell function in a model of beta cell-specific deletion of PC2 and human proIAPP expression.

Whether impaired proIAPP is characteristic of type 2 diabetes has not been determined; however, elevated proinsulin:insulin ratios in circulation have been shown to be markers of beta cell dysfunction in type 1 diabetes [35], type 2 diabetes [15, 18] and islet transplant failure [18]. In this study, we present an in vivo model in which impaired processing of human proIAPP exacerbates islet transplant failure, speculating that it may also contribute to diabetes. Together, these findings may implicate IAPP precursors as cytotoxic species in the beta cell and as potential biomarkers of beta cell dysfunction.

Acknowledgements We thank P. Y. T. Lu for careful editing of the manuscript and K. Song for assistance in histology quantification (both from the Research Institute, BC Children's Hospital, Vancouver, BC, Canada), and the Canucks for Kids Fund Childhood Diabetes Laboratories for core support.

Funding This work was supported by grants to CBV from the Canadian Institutes of Health Research (CIHR; MOP-14682) and the Canadian Diabetes Association (OG-3-11-3413-CV). CBV was supported by an investigator award from BC Children's Hospital and the Irving K. Barber Chair in Diabetes Research. JAC was supported by a trainee award from the CIHR Transplant Training Program.

Duality of interest The authors declare that there is no duality of interested associated with their contribution to this manuscript.

Data availability The data that support the findings of this study are available from the corresponding author upon reasonable request.

Contribution statement JAC, PCO and CBV contributed to the design and conceptualised the experiments described. JAC, EYW, PY, DLD and GS conducted the experiments and analysed the data. JAC and CBV wrote the manuscript. All authors performed critical analysis of the manuscript and approved the final version to be published. CBV is the guarantor of this work.

\section{References}

1. Butler AE, Janson J, Bonner-Weir S, Ritzel R, Rizza RA, Butler PC (2003) $\beta$-Cell deficit and increased $\beta$-cell apoptosis in humans with type 2 diabetes. Diabetes 52:102-110

2. Kahn SE, D'Alessio DA, Schwartz MW et al (1990) Evidence of cosecretion of islet amyloid polypeptide and insulin by $\beta$-cells. Diabetes 39:634-638

3. Stridsberg M, Sandler S, Wilander E (1993) Cosecretion of islet amyloid polypeptide (IAPP) and insulin from isolated rat pancreatic islets following stimulation or inhibition of beta-cell function. Regul Pept 45:363-370

4. Chargé SBP, Esiri MM, Bethune CA, Hansen BC, Clark A (1996) Apolipoprotein $\mathrm{E}$ is associated with islet amyloid and other amyloidoses: implications for Alzheimer's disease. J Pathol 179:443447

5. Pepys MB, Rademacher TW, Amatayakul-Chantler S et al (1994) Human serum amyloid $\mathrm{P}$ component is an invariant constituent of amyloid deposits and has a uniquely homogeneous glycostructure. Proc Natl Acad Sci U S A 91:5602-5606

6. Abedini A, Tracz SM, Cho JH, Raleigh DP (2006) Characterization of the heparin binding site in the N-terminus of human pro-islet amyloid polypeptide: implications for amyloid formation. Biochemistry 45:9228-9237

7. Lin CY, Gurlo T, Kayed R et al (2007) Toxic human islet amyloid polypeptide (h-IAPP) oligomers are intracellular, and vaccination to induce anti-toxic oligomer antibodies does not prevent h-IAPPinduced $\beta$-cell apoptosis in h-IAPP transgenic mice. Diabetes 56: $1324-1332$

8. Kayed R (2003) Common structure of soluble amyloid oligomers implies common mechanism of pathogenesis. Science 300:486489

9. Masters SL, Dunne A, Subramanian SL et al (2010) Activation of the NLRP3 inflammasome by islet amyloid polypeptide provides a mechanism for enhanced IL- $1 \beta$ in type 2 diabetes. Nat Immunol 11:897-904

10. Westwell-Roper C, Dai DL, Soukhatcheva G et al (2011) IL-1 blockade attenuates islet amyloid polypeptide-induced proinflammatory cytokine release and pancreatic islet graft dysfunction. J Immunol 187:2755-2765

11. Westwell-Roper CY, Ehses JA, Verchere CB (2014) Resident macrophages mediate islet amyloid polypeptide-induced islet IL-1 $\beta$ production and $\beta$-cell dysfunction. Diabetes 63:1698-1711

12. Potter KJ, Abedini A, Marek P et al (2010) Islet amyloid deposition limits the viability of human islet grafts but not porcine islet grafts. Proc Natl Acad Sci U S A 107:4305-4310

13. Udayasankar J, Kodama K, Hull RL et al (2009) Amyloid formation results in recurrence of hyperglycaemia following transplantation of human IAPP transgenic mouse islets. Diabetologia 52:145153

14. Matveyenko AV, Butler PC (2006) $\beta$-cell deficit due to increased apoptosis in the human islet amyloid polypeptide transgenic (HIP) rat recapitulates the metabolic defects present in type 2 diabetes. Diabetes 55:2106-2114

15. Ward WK, LaCava EC, Paquette TL, Beard JC, Wallum BJ, Porte D Jr (1987) Disproportionate elevation of immunoreactive proinsulin in Type 2 (non-insulin-dependent) diabetes mellitus and in experimental insulin resistance. Diabetologia 30:698-702

16. Nagi DK, Ali VM, Yudkin JS (1996) Effect of metformin on intact proinsulin and des 31,32 proinsulin concentrations in subjects with non-insulin-dependent (Type 2) diabetes mellitus. Diabet Med 13: 753-757

17. Zhu X, Orci L, Carroll R, Norrbom C, Ravazzola M, Steiner DF (2002) Severe block in processing of proinsulin to insulin 
accompanied by elevation of des-64,65 proinsulin intermediates in islets of mice lacking prohormone convertase 1/3. Proc Natl Acad Sci U S A 99:10299-10304

18. Klimek AM, Soukhatcheva G, Thompson DM et al (2009) Impaired proinsulin processing is a characteristic of transplanted islets. Am J Transplant 9:2119-2125

19. Zhu X, Zhou A, Dey A et al (2002) Disruption of PC1/3 expression in mice causes dwarfism and multiple neuroendocrine peptide processing defects. Proc Natl Acad Sci U S A 99:10293-10298

20. Furuta M, Yano H, Zhou A et al (1997) Defective prohormone processing and altered pancreatic islet morphology in mice lacking active SPC2. Proc Natl Acad Sci 94:6646-6651

21. Furuta M, Carroll R, Martin S et al (1998) Incomplete processing of proinsulin to insulin accompanied by elevation of Des-31,32 proinsulin intermediates in islets of mice lacking active PC2. J Biol Chem 273:3431-3437

22. Yu SS, Kitbachi AE (1973) Biological activity of proinsulin and related polypeptides in the fat tissue. J Biol Chem 248:3753-3761

23. Marzban L, Trigo-Gonzalez G, Zhu X et al (2004) Role of $\beta$-cell prohormone convertase (PC) $1 / 3$ in processing of pro-islet amyloid polypeptide. Diabetes 53:141-148

24. Wang J, Xu J, Finnerty J, Furuta M, Steiner DF, Verchere CB (2001) The prohormone convertase enzyme 2 (PC2) is essential for processing pro-islet amyloid polypeptide at the NH2-terminal cleavage site. Diabetes 50:534-539

25. Exley C, House E, Patel T, Wu L, Fraser PE (2010) Human pro-islet amyloid polypeptide (ProIAPP(1-48)) forms amyloid fibrils and amyloid spherulites in vitro. J Inorg Biochem 104:1125-1129

26. Khemtémourian L, Casarramona GL, Suylen DPL et al (2009) Impaired processing of human pro-islet amyloid polypeptide is not a causative factor for fibril formation or membrane damage in vitro. Biochemistry 48:10918-10925

27. Farooqi IS, Volders K, Stanhope R et al (2007) Hyperphagia and early-onset obesity due to a novel homozygous missense mutation in prohormone convertase 1/3. J Clin Endocrinol Metab 92:33693373

28. Paulsson JF, Westermark GT (2005) Aberrant processing of human proislet amyloid polypeptide results in increased amyloid formation. Diabetes 54:2117-2125

29. Marzban L, Rhodes CJ, Steiner DF, Haataja L, Halban PA, Verchere CB (2006) Impaired NH2-terminal processing of human proislet amyloid polypeptide by the prohormone convertase PC2 leads to amyloid formation and cell death. Diabetes 55:2192-2201

30. Alarcon C, Verchere CB, Rhodes CJ (2012) Translational control of glucose-induced islet amyloid polypeptide production in pancreatic islets. Endocrinology 153:2082-2087

31. Meng F, Abedini A, Song B, Raleigh DP (2007) Amyloid formation by pro-islet amyloid polypeptide processing intermediates: examination of the role of protein heparan sulfate interactions and implications for islet amyloid formation in type 2 diabetes. Biochemistry 46:12091-12099

32. Potter KJ, Werner I, Denroche HC et al (2015) Amyloid formation in human islets is enhanced by heparin and inhibited by heparinase. Am J Transplant 15:1519-1530

33. Park K, Verchere CB (2001) Identification of a heparin binding domain in the N-terminal cleavage site of pro-islet amyloid polypeptide. Implications for islet amyloid formation. J Biol Chem 276: 16611-16616

34. Webb GC, Akbar MS, Zhao C, Swift HH, Steiner DF (2002) Glucagon replacement via micro-osmotic pump corrects hypoglycemia and $\alpha$-cell hyperplasia in prohormone convertase 2 knockout mice. Diabetes 51:398-405

35. Truyen I, De Pauw P, Jørgensen PN et al (2005) Proinsulin levels and the proinsulin:C-peptide ratio complement autoantibody measurement for predicting type 1 diabetes. Diabetologia 48:23222329 